\title{
Representation of Accurate Temporal Information in the Electrosensory System of the African Electric Fish, Gymnarchus niloticus
}

\author{
Yuan-Xing Guo and Masashi Kawasaki \\ Department of Biology, University of Virginia, Charlottesville, Virginia 22903
}

Differential-phase-sensitive neurons in the electrosensory lateral line lobe (ELL) of the African electric fish, Gymnarchus niloticus, are sensitive to time disparities on the order of microseconds between afferent action potentials. These action potentials fire in a phase-locked manner in response to the animal's own wave-type electric organ discharges (EODs) (Kawasaki and Guo, 1996). The time disparity is one of the essential cues for an electrical behavior, the jamming avoidance response (JAR). To gain an insight into the accurate temporal processing in the ELL, firing time accuracy and dynamic response properties of action potentials of the phaselocked neurons (PLNs) in the ELL were examined. The temporal accuracy of the entire neuronal circuit for the JAR was also measured using behavioral responses.

Standard deviation of firing times of PLNs' action potentials was $\sim 6 \mu \mathrm{sec}$. The PLNs represent zerocrossing times of each stimulus cycle with this accuracy even when stimulus phase was modulated at high frequencies $(\sim 50 \mathrm{~Hz})$. Distinct JAR occurred when time disparity was diminished below $1 \mu \mathrm{sec}$, and a marginal JAR could still be detected with a time disparity of $100 \mathrm{nsec}$. Standard deviation of the firing times of EODs was approximately several hundred nanoseconds. This stability of the EOD, however, was demonstrated to be unnecessary for the JAR. JARs occurred even when a large artificial jitter $(\sim 60$ $\mu \mathrm{sec})$ was introduced to a stimulus that mimicked fish's own EOD and the time disparity for JAR was diminished to $1 \mu \mathrm{sec}$. This immunity of JAR to the EOD jitter is explained by the insensitivity of the differential-phase-sensitive neurons in the ELL to a common phase modulation.

The JAR of the South American electric fish, Eigenmannia, also occurs in response to stimuli that generate comparably small phase differences (Rose and Heiligenberg, 1985b; Carr et al., 1986a). The present study revealed that the independently evolved Eigenmannia and Gymnarchus exhibit a comparative level of remarkable temporal accuracy.

Key words: phase; phase-locked neurons; electric fish; jamming avoidance response; phase comparison; binaural comparison; convergent evolution; adaptation; vector strength
Behavioral studies of the auditory system indicate that temporal disparities on the order of microseconds can be resolved by the CNS. Human subjects can localize a sound source with an accuracy of $\sim 1^{\circ}$ using small differences in sound arrival times between two ears (Mills, 1972). Several species of vertebrate animals also use small interaural time differences to localize sound sources (Rabizza and Masterton, 1972; Brown et al., 1978; Knudsen et al., 1979; Simmons et al., 1983). Although such behavioral experiments clearly demonstrate the ability of the CNS to deal with temporal codes of microseconds or even submicroseconds, the physiological mechanisms underlying this capability are not fully understood.

Kawasaki and Guo (1996) recently discovered that neurons in an early stage of central processing of electrosensory information in an African electric fish, Gymnarchus niloticus, exhibit a sensitivity to time disparities between electrosensory inputs on the order of microseconds.

A "wave"-type electric fish, Gymnarchus, constantly emits cyclic electric organ discharges (EODs) at individually fixed frequencies

Received Sept. 30, 1996; revised Nov. 12, 1996; accepted Dec. 9, 1996.

This study was supported by a National Institute of Mental Health Grant R29 MH48115-01A1 and by a Research Scientist Development Award K-02 MH01256-01 from ADAMHA to M.K. We thank Yasuko Kawasaki for figure preparation, two anonymous referees for critical comments, and Cameron McLaughlin for editing English.

Correspondence should be addressed to Masashi Kawasaki, Department of Biology, Gilmer Hall, University of Virginia, Charlottesville, VA 22903.

Copyright (C) 1997 Society for Neuroscience $0270-6474 / 97 / 171761-08 \$ 05.00 / 0$
$(300-400 \mathrm{~Hz})$. An electric field is thus established around the body, and its distortion by objects is detected by electroreceptors located over the body surfaces (Lissmann and Machin, 1958). When two fish with similar discharge frequencies meet, they shift their discharge frequencies away from each other to avoid mutual jamming of their electrolocation systems (Bullock et al., 1975). This jamming avoidance response (JAR) requires the detection of time disparities between sensory signals at different electroreceptors (Kawasaki, 1993a). The signal phases, or zerocrossing times of the electrosensory signal received by electroreceptors, are conveyed in a phase-locked manner to the first brain station, the electrosensory lateral line lobe (ELL), by S-type afferent fibers. While directly projecting to the inner cell layer (ICL) of the ELL, collateral axons of the S-type afferents synapse onto the giant cells of the ELL, which in turn project to the ICL. Because the S-type afferent fibers and giant cells, collectively referred to as phaselocked neurons (PLNs) in this study, fire one action potential for each zerocrossing of the electrosensory signal with a certain latency, times of zerocrossing of signals at different electroreceptors are represented at the ICL as the times of the action potentials of PLNs. Kawasaki and Guo (1996) describe physiological and morphological characteristics of these PLNs that give input to differential-phase-sensitive neurons that are sensitive to time disparities on the order of single-digit microseconds (Fig. 1).

Possible physiological requirements for such neuronal sensitivity to small time disparities include accurate representation of temporal information by PLNs that provide synaptic input to the 
Figure 1. Transverse section of the ELL of Gymnarchus showing the neuronal circuit for phase comparison. S-type afferent fibers ipsilaterally project to the inner cell layer $(I C L)$ of the ELL. Axon collaterals of S-type afferents project to the adendritic soma of the giant cells which, in turn, bilaterally project also to the ICL. In the ICL are differentialphase-sensitive neurons that receive inputs from the terminals of S-type afferents and giant cells. Based on camera lucida drawings of biocytin-filled neurons.

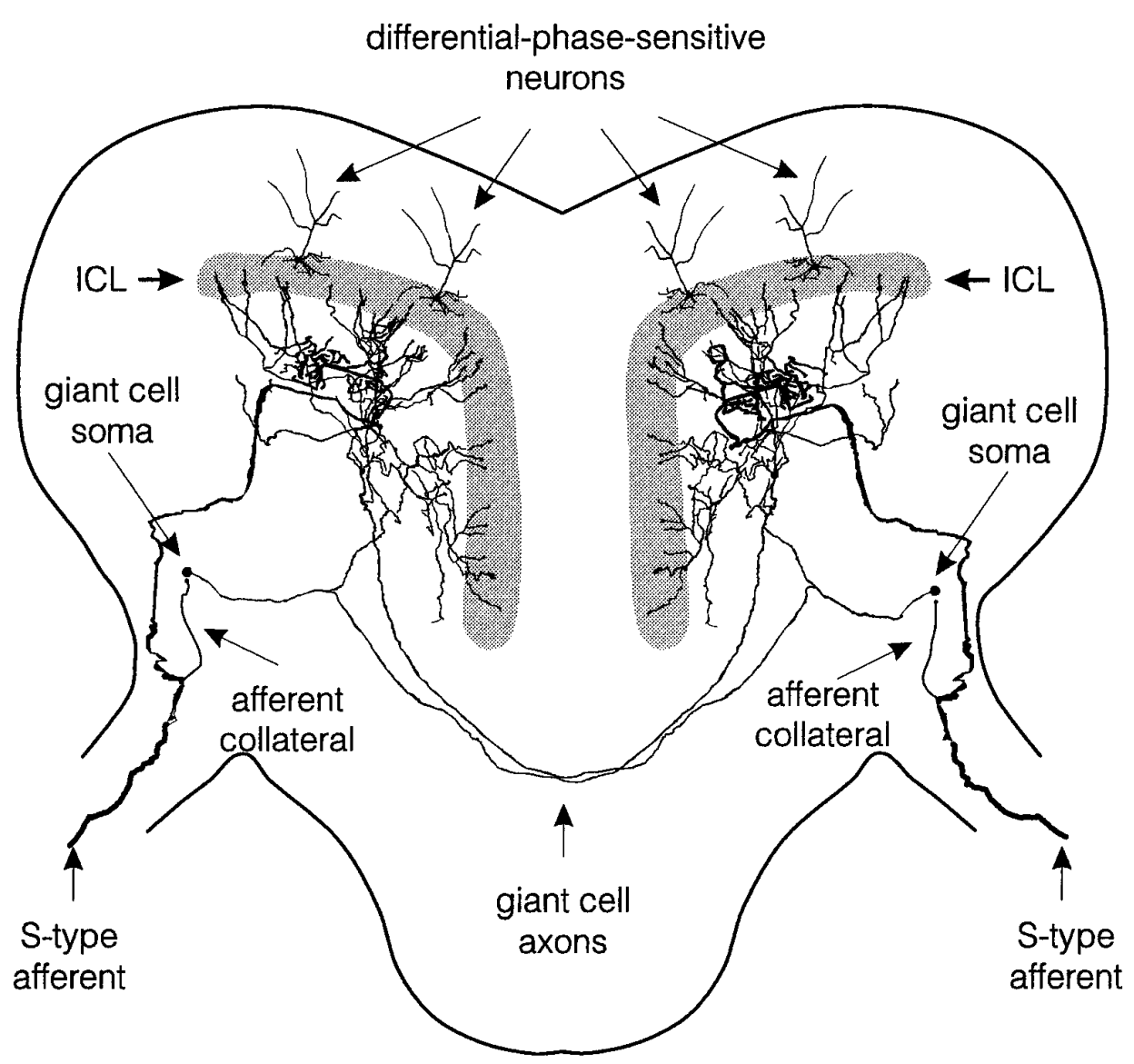

differential-phase-sensitive neurons. In this study, temporal accuracy and dynamics of individual PLNs in the ELL were examined. Standard deviation of firing times, or jitter, of PLNs was as small as $5.6 \mu \mathrm{sec}$ on average.

Expression of this neuronal accuracy for temporal processing in behavioral output was also examined by measuring thresholds for time disparities for the JAR. Gymnarchus was found to exhibit JARs in response to stimulus patterns capable of generating time disparities among electroreceptor afferents of only a few hundred nanoseconds.

These results were compared with those obtained previously in an independently evolved gymnotiform electric fish, Eigenmannia, which performs the same type of JARs (Rose and Heiligenberg, 1985b; Carr et al., 1986a,b).

\section{MATERIALS AND METHODS}

Animals and physiological recording. Approximately 50 Gymnarchus niloticus $(13-20 \mathrm{~cm})$ were used. Environmental conditions in the holding tanks were identical to those described in Kawasaki (1994). For both physiological and behavioral experiments, intramuscular injection of Flaxedil (gallamine triethiodide, $0.03 \%, 3-8 \mu \mathrm{l}$ ) immobilized fish and greatly attenuated EOD amplitude. Oxygen-saturated water was provided through a mouthpiece to maintain the immobilized preparation.

After local application of Xylocane (2\%), a small hole was drilled in the skull above the corpus cerebelli. The caudal part of the ELL was exposed by removing the caudal edge of the corpus cerebelli with fine suction tubing (inner diameter $500 \mu \mathrm{m}$ ). Fish were gently held with a spongelined clamp and submerged in water (tank size $\sim 40 \times 40 \times 10 \mathrm{~cm}$ ) except for a small area around the skull opening. Intracellular recordings from PLNs were made with sharp glass-capillary electrodes (30-50 M $\Omega, 3 \mathrm{M}$ $\mathrm{KCl}$ ) in the deep fiber layer of the ELL (Bass and Hopkins, 1982). This layer was localized by recording field potentials in the ELL that show systematic change of waveform along the depth (see Fig. 2 in Kawasaki and Guo, 1996) with a glass-capillary electrode (5-10 $\mu \mathrm{m}$ tip diameter, 3 $\mathrm{M} \mathrm{NaCl}$ ). No anatomical confirmation was made to distinguish recordings from the terminals of the S-type afferent fibers and giant neurons.

Attenuated EODs were recorded in the immobilized preparation to register JAR by suction electrode attached to the tail. EODs were also measured in intact fish that were freely swimming but voluntarily hiding in a plastic tubing (diameter $5 \mathrm{~cm}$; length $15 \mathrm{~cm}$, slightly shorter than that of the fish). Recording electrodes for EODs were attached at both ends of the tubing.

Stimulus presentation. A sinusoidal signal, $\mathrm{S}_{1}$, which simulated the fish's own EOD, was applied between a silver wire electrode in the mouth and an electrode near the tail. This electrode pair simulated the amplitude and geometry of the electric field generated by the fish's own EODs before being silenced with Flaxedil. $\mathrm{S}_{1}$ was generated by a phase synthesizer (Wavetek, 650) with a phase accuracy of $35 \mathrm{nsec}$. The frequency of $S_{1}$ was set constant to be near that of fish's EOD before immobilization. For measuring dynamic phase responses, $S_{1}$ was sinusoidally modulated in phase by applying a sinusoidal signal to the phase-modulation input of the phase synthesizer. To add artificial jitter in $S_{1}$, noise was generated with a white-noise generator (Hewlett Packard, HP-8057A), filtered (Krohn Hite, 3550R, passband 20-200 Hz), and fed into the phase modulation port of the phase synthesizer. The phase modulation of $S_{1}$ by the noise was measured and expressed by the same method as for measuring neuronal jitter (see below).

To induce JAR, a sinusoidal signal, $\mathrm{S}_{2}$, which simulated the EOD of a neighboring fish, was applied through an independent electrode pair straddling the fish. The frequency of $S_{2}$ was switched every $30 \mathrm{sec}$ between $2 \mathrm{~Hz}$ higher and $2 \mathrm{~Hz}$ lower than that of $\mathrm{S}_{1}$. A 4 min session consisted of eight alternating $30 \mathrm{sec}$ periods of frequency differences, +2 and $-2 \mathrm{~Hz}$. The magnitude of JAR was measured as an average of responses during these eight consecutive periods. For each period, timings of every 100 EODs were registered with a divide-by-100 counter (see below). Thus, EOD frequency was averaged over $\sim 250 \mathrm{msec}$ and sampled $\sim 4$ times $/ \mathrm{sec}$. The change in frequency was integrated over each of the $30 \mathrm{sec}$ periods and converted to the unit of $\mathrm{Hz} / \mathrm{min}$. The sign of the frequency shift in each period was defined as positive when JAR occurred in the expected 
direction and as negative when frequency shift occurred in the opposite direction. Percentage of correct responses was calculated as the number of periods in which a positive frequency shift occurred by dividing by 8 . The sign test shows that $100 \%(8 / 8)$ and $87.5 \%(7 / 8)$ correct responses correspond to $p=0.0039$ and $p=0.0351$, respectively. Cases of $<75 \%$ $(6 / 8, p=0.14)$ are all statistically insignificant. The threshold for the JAR was determined by attenuating $S_{2}$ with a custom-made signal attenuator the $\mathrm{S} / \mathrm{N}$ ratio of which was $>100 \mathrm{~dB}$.

Measurement and expression of phase. Intracellular potentials of PLNs were recorded with sharp glass-capillary electrodes filled with $3 \mathrm{M} \mathrm{KCl}$ that were connected to an electrometer (WPI, S-7071A). Recording noise was $<0.5 \mathrm{mV}$. When two PLNs were recorded simultaneously, they were from one side of the ELL. The capacitive compensation circuit in the electrometer was turned off because it introduced a high-frequency $(>10$ $\mathrm{kHz}$ ) noise that disrupted accurate measure of zerocrossing times. Intracellular potentials were amplified by $60 \mathrm{~dB}$ with an AC-coupled operational amplifier (TL074) specially installed within the electrometer. This amplified signal was AC-coupled further, clipped at $\pm 0.7 \mathrm{~V}$ by diodes, and sent to a custom-made Schmitt trigger circuit that compared input signals against a stable reference of $0 \mathrm{~V}$ and generated TTL pulses associated with zerocrossings. The $60 \mathrm{~dB}$ amplification greatly eliminated artifact jitter associated with the Schmitt triggering of high-frequency noise components introduced in the recording and reference signal. This notion was supported by the fact that further amplification $(80 \mathrm{~dB})$ did not improve jitter measure. EODs recorded from intact fish were also amplified by $60 \mathrm{~dB}$ before being sent to the Schmitt trigger circuit.

Successive positive zerocrossing times, $t_{i}$, of action potentials of PLNs were registered by a Schmitt trigger circuit of a time-stamp recorder (Tucker-Davis Technology, ET-1) with a resolution of 100 nsec. Two time-stamp recorders were used to measure phase differences between a stimulus and action potentials of a PLN, or between two PLNs. These recorders were commonly driven by a single crystal oscillator to ensure an accurate measure of phase differences.

A successive series of phases, $p_{i}$, is expressed in $\mu$ sec as:

$$
p_{i}=t_{i}-r_{i},
$$

where $t_{i}$ are zerocrossing times of action potentials and $r_{i}$ (reference times) are the predicted zerocrossing times of an ideally constant signal with no jitter. $r_{i}$ values were obtained either by hardware synchronization pulses from the stimulus generator or by computing with sufficient accuracy as:

$$
r_{i}=\frac{t_{n}-t_{1}}{n-1} \times(i-1),
$$

where $n$ is the number of time stamps, which was typically $\sim 3500$ for the standard $10 \mathrm{sec}$ sampling period. These two methods yielded indistinguishable $r_{i}$ because the hardware synchronization pulses from the signal generator and the computation were equally accurate $(<35 \mathrm{nsec})$.

Standard deviation, amplitude component of Fourier spectrum, time series, and its probability distribution function of $p_{i}$ were computed and plotted by a 486 PC (Gateway 2000, DX486/33) using custom software.

Jitter of EOD was measured by a similar method. As for PLNs, EOD was amplified by $60 \mathrm{~dB}$ and sent to the same time-stamp recorder, which produced a series of time stamps, $t_{i}$. A series of periods, $q_{i}$, was computed as:

$$
q_{i}=t_{i}-t_{i-1} .
$$

Gymnarchus often showed a very slow drift in EOD frequency that yielded a continuous change of $q_{i}$. When this happened, measurement was halted until the fish stopped such drift.
Standard deviation $(\sigma)$ of $p_{i}$ and $q_{i}$ is hereafter referred to as jitter.

Estimation of phase difference between PLNs during the JAR. Magnitude of phase modulation of the signal mixture of $\mathrm{S}_{1}$ and $\mathrm{S}_{2}$ is a function of the amplitude ratio of $S_{1}$ and $S_{2},\left|S_{2}\right| /\left|S_{1}\right|$ (Heiligenberg and Bastian, 1980a; Kawasaki, 1993b). The magnitude is computed as:

$$
\phi \approx\left|\mathrm{S}_{2}\right| /\left|\mathrm{S}_{1}\right| \times T / 2 \pi,
$$

where $T$ is the period of $\mathrm{S}_{1}$. $\phi$, however, differs across the body areas because of different $\left|S_{2}\right| /\left|S_{1}\right|$ ratios at different areas. $\left|S_{2}\right| /\left|S_{1}\right|$ ratios are different because electric field vectors by fish's own and neighbor's EOD form different angles at different body areas. These differences in $\phi$ create phase differences between body areas, which are one of the essential cues for the JAR (see Fig. 3 in Kawasaki, 1993b). Therefore, an $\left|S_{2}\right| /\left|S_{1}\right|$ ratio measured at a particular point on the body surface does not give an estimate of the magnitude of phase modulation for individual PLNs that represent different areas of the body surface. Distribution of the magnitude of phase modulation of PLNs at a given stimulus condition was examined by recording intracellularly from 220 PLNs in the ELL while $\left|\mathrm{S}_{2}\right| /\left|\mathrm{S}_{1}\right|$ was set to 0.2 as measured at a standard location, the gill cover. There $\left|S_{2}\right| /\left|S_{1}\right|$ was measured by a pair of fork electrodes $(1 \mathrm{~cm}$ separation), one pole of which was placed close to the gill cover. The two poles of the pair were oriented at the angle perpendicular to the gill cover. The frequency difference between $S_{1}$ and $S_{2}$ was set to $2 \mathrm{~Hz}$. Magnitudes of phase modulation in PLN action potentials, which occurred at $2 \mathrm{~Hz}$ in this condition, were computed from the $2 \mathrm{~Hz}$ component of the amplitude spectrum of $p_{i}$. Mean, standard deviation, and the minimum and maximum of the magnitude of phase modulation of 220 PLNs were 16, 10, 0.3, and $48 \%$, respectively, of the estimated magnitude from the ratio $\left|\mathrm{S}_{2}\right| /\left|\mathrm{S}_{1}\right|$ at the standard location by Equation 4. "Mean phase modulation" in this study is defined as estimated modulation depths by Equation 4 multiplied by $16 \%$. The high estimate of $\left|S_{2}\right| /\left|S_{1}\right|$ measured at the standard location is probably because $S_{1}$ current density rapidly falls with the distance from the gill cover behind which one of the current sources was located, and the pair of recording electrodes with $1 \mathrm{~cm}$ separation did not effectively measure the local current density at the surface of the gill cover. Because the right and left sides experience phase modulations that are $180^{\circ}$ out of synchronization (see Fig. 10 in Heiligenberg and Rose, 1985), mean phase differences between the two sides available to the differentialphase-sensitive neurons in the ELL are twice as large as "mean phase modulation."

\section{RESULTS}

\section{Accuracy of phase representation by PLNs}

We sampled $p_{i}$ of intracellularly recorded action potentials from 134 PLNs in the ELL (Fig. 2). Figure $2 A$ shows $p_{i}$ of action potentials from a representative PLN whose jitter was $7.8 \mu \mathrm{sec}$. Jitters of 134 PLNs were tightly distributed around the mean of $6.5 \mu \mathrm{sec}$. A jitter of $<10 \mu \mathrm{sec}$ was shown in $95 \%$ of recorded PLNs, and the most accurate PLN showed a jitter of $1.3 \mu \mathrm{sec}$ (Fig. 2B). The frequency contents of $p_{i}$ shown by these neurons were examined by Fourier analysis. As shown in Figure $2 C$, the amplitude spectrum of $p_{i}$ was flat except for a prominent peak at $60 \mathrm{~Hz}$. The $60 \mathrm{~Hz}$ peak was seen in all cases studied and is believed to be the power line frequency contamination through the recording system. Thus, a neuronal noise with a wide range of frequencies contributed to the jitter. To

Table 1. Correlation of jitter in 40 pairs of simultaneously recorded PLNs

\begin{tabular}{llccr}
$\sigma$ of $\left\{P A_{i}\right\}$ & $\begin{array}{l}\sigma \text { of }\left\{P B_{i}\right\} \\
(\mu \mathrm{sec})\end{array}$ & $\begin{array}{l}\sigma \text { of }\left\{P A_{i}-P B_{i}\right\} \\
(\mu \mathrm{sec})\end{array}$ & $\begin{array}{r}\text { Correlation coefficient } \\
\text { between }\left\{P A_{i}\right\} \text { and }\left\{P B_{i}\right\}\end{array}$ \\
\hline Mean & 4.50 & 4.35 & 5.99 & 0.15 \\
SD & 2.75 & 1.91 & 2.32 & 0.17 \\
Minimum & 1.34 & 1.00 & 1.47 & -0.18 \\
Maximum & 14.28 & 9.78 & 10.65 & 0.61 \\
$n$ & 40 & 40 & 40 & 40 \\
\hline
\end{tabular}

$\left\{P A_{i}\right\}$ and $\left\{P B_{i}\right\}$ are the time series of phase defined as Equation 1 for neurons $\mathrm{A}$ and $\mathrm{B}$, respectively, which were simultaneously recorded as a pair. 
A
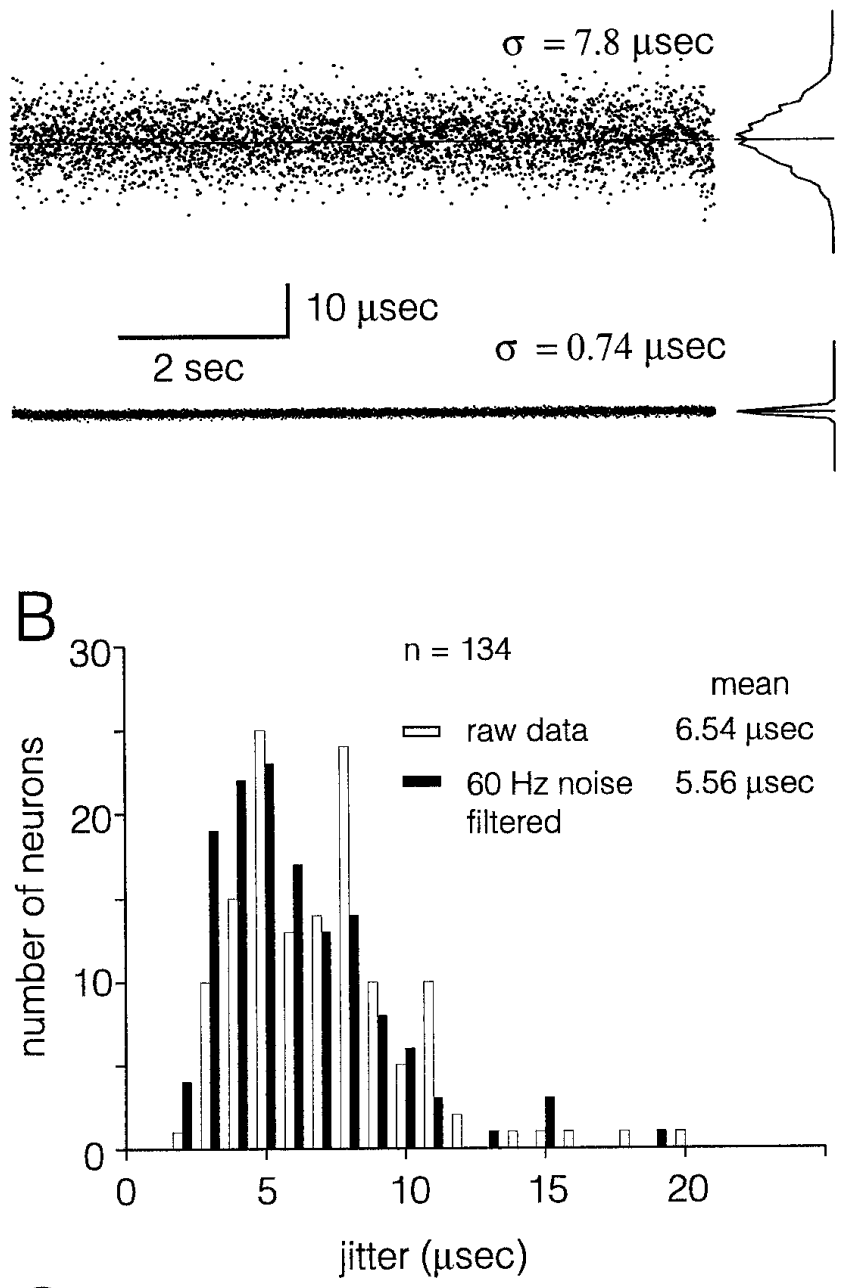

C

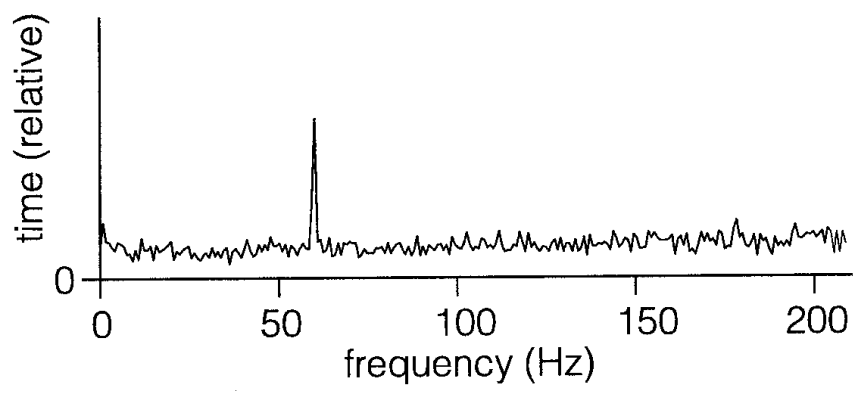

Figure 2. Jitter of action potentials of PLNs in the ELL in response to unmodulated $\mathrm{S}_{1}$. A, Left, A time series plot of $p_{i}$ of a representative PLN (top) and $\mathrm{S}_{1}$ measured in the experimental tank (bottom). Right, Probability distribution function of the time series plots on the left. The bottom trace was obtained by recording $\mathrm{S}_{1}$ using the same amplifier and recording system as for the intracellular recording in the top trace. $B$, Distribution of jitter, or SD $(\sigma)$ in 134 PLNs. Open bars represent data from raw $p_{i}$; filled bars represent data from $p_{i}$ processed by a $60 \mathrm{~Hz}$ digital filter (see Results). $C$, Amplitude spectrum of $p_{i}$ in a representative PLN. Note the generally flat spectrum with a sharp peak at $60 \mathrm{~Hz}$, which is an artifact of power line contamination. SDs of $p_{i}$ before and after the digital filtering were 7.1 and $4.9 \mu \mathrm{sec}$, respectively, in this particular PLN. assess the contribution of the $60 \mathrm{~Hz}$ peak to jitter magnitude, $p_{i}$ were digitally filtered against $60 \mathrm{~Hz}$ and $p_{i}$ was reconstructed by reverse FFT. This procedure reduced the mean of standard deviations by $14 \%$, yielding $5.6 \mu \mathrm{sec}$ (Fig. $2 B$ ).

\section{Jitter correlation between PLNs}

Jitter in individual PLNs could be caused by the following: (1) noise commonly affecting all PLNs, such as inherent noise in $S_{1}$ and noise in electroreceptors that may be coupled to each other (Szabo, 1962); and (2) noise arising independently in individual PLNs. Differential-phase-sensitive neurons in the ELL, which detect phase differences between inputs from PLNs, must be affected by the latter type of noise but may be immune to the noise common in all PLNs. To investigate differential jitter between PLNs, we made simultaneous intracellular recordings from 40 pairs of PLNs. Jitter in individual neurons, jitter of phase differences between the two PLNs, and correlation coefficients between $p_{i}$ of the two neurons are presented in Table 1. Correlation coefficients were small, indicating that a major source of jitter can be attributed to a noise arising independently in different PLNs.

\section{Dynamic responses of PLNs}

We examined dynamics of the PLNs by modulating the phase of $\mathrm{S}_{1}$ at different frequencies and measured the dynamic responses of the resulting phase modulation in PLNs (Fig. 3). All neurons responded at near unity gain from 0.4 to $15 \mathrm{~Hz}$. Gain was reduced to $0.5 \pm 0.12$ at $100 \mathrm{~Hz}$ (Fig. 3C). Even with a large modulation $(50 \mu \mathrm{sec})$, the accuracy with which the PLN action potentials encoded stimulus phase was preserved, as shown in the jitter of the top traces in Figure 3, $A$ and $B$. Although gain was constant up to $15 \mathrm{~Hz}$, different neurons may have shown different phase delay, which may have introduced phase differences among PLNs. To examine this possibility, we recorded simultaneously from two PLNs and measured phase differences during sinusoidal modulations of $\mathrm{S}_{1}$ phase at different frequencies. As shown in Figure 3, D and $E$, two neurons sampled simultaneously were phasemodulated in synchrony, leaving only small phase differences even at high modulation frequencies $(\sim 100 \mathrm{~Hz})$. These data indicate that amplitude and time course of phase modulation in $S_{1}$ are accurately represented by PLNs from low frequencies up to a few tens of Hertz.

\section{Behavioral threshold for the JAR}

The limit of Gymnarchus' ability to detect small phase differences was tested by measuring JAR under conditions in which phase difference was progressively reduced. The phase-chamber stimulus delivery method (Rose and Heiligenberg, 1985b; Kawasaki, 1993a,b), in which body surface was electrically divided into two sections, provides phase differences with a known minimum value. Although this method yielded robust JARs when phase differences exceeded tens of microseconds, it failed to evoke any detectable JAR when phase differences fell below $10 \mu$ sec in our preliminary experiments. The free-field stimulus delivery method (Kawasaki, 1993a), in which $S_{1}$ and $S_{2}$ were mixed in the experimental tank, however, was much more effective in evoking JAR when phase differences were smaller. Therefore, the free-field condition was used to measure the behavioral threshold for phase differences. Phase differences were estimated as described in Materials and Methods. Figure $4 A$ shows JAR measured under progressively smaller phase modulations. Figure $4 B$ shows percent correct responses with various mean phase modulations from eight individuals. JAR always occurred in the correct direction with mean phase modulation of $>1 \mu$ sec. When mean phase 

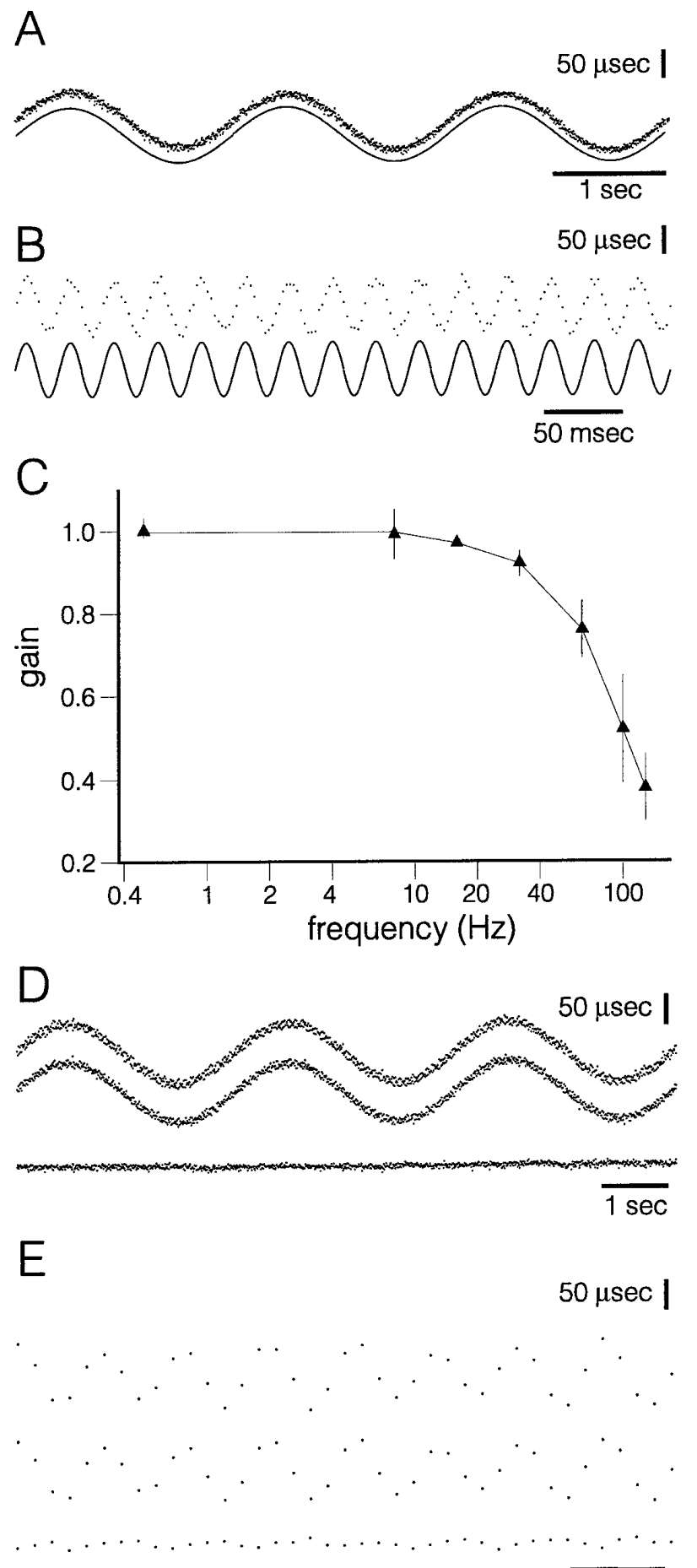

$\overline{10 \mathrm{msec}}$

Figure 3. Magnitude of phase modulation of PLNs in response to $\mathrm{S}_{1}$, the phase of which was sinusoidally modulated at different frequencies. $A, \mathrm{~S}_{1}$ was phase-modulated by $\pm 50 \mu \mathrm{sec}$ at $0.5 \mathrm{~Hz}$ (solid sinusoids at bottom), and the magnitude of phase modulation in response (dots at top) was measured by a $0.5 \mathrm{~Hz}$ Fourier component of the top traces. B, Same experiment except that the modulation frequency was $32 \mathrm{~Hz}$. $C$, Gain of phase responses in PLNs for different modulation frequencies. Gain was computed as the ratio between the depths of modulation in the stimulus and the response. Each data point represents mean \pm SD from at least 14 PLNs. D, E, Simultaneous recordings from two PLNs (top and middle) and phase difference between them (bottom). Phase modulation in stimuli was $\pm 50 \mu \mathrm{sec}$ in both cases; modulation frequencies in $D$ and $E$ were 0.4 and $100 \mathrm{~Hz}$, respectively.
A
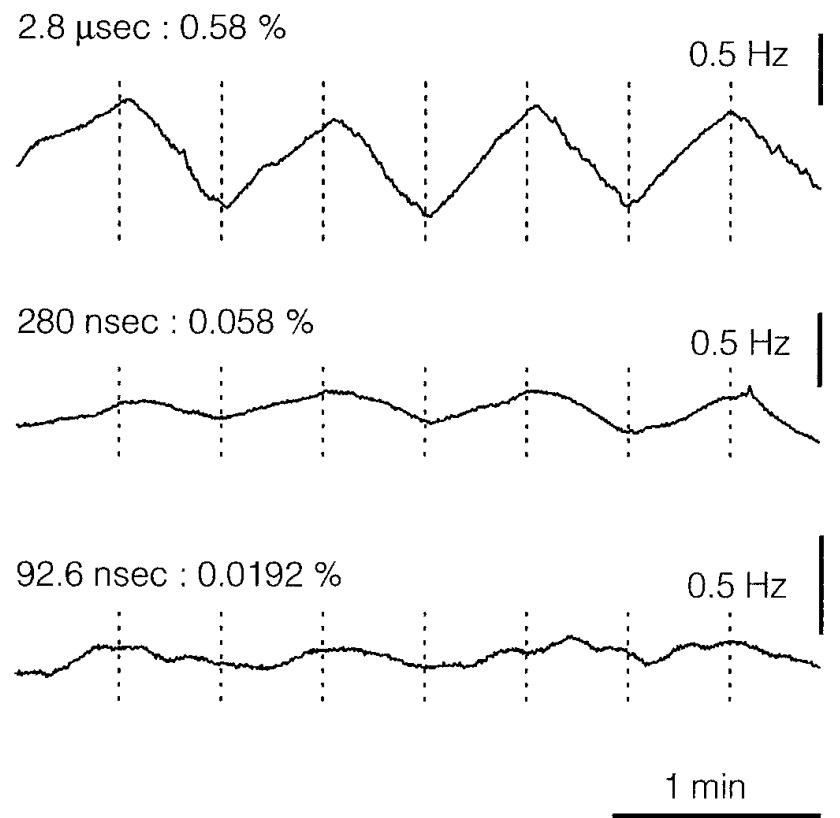

B

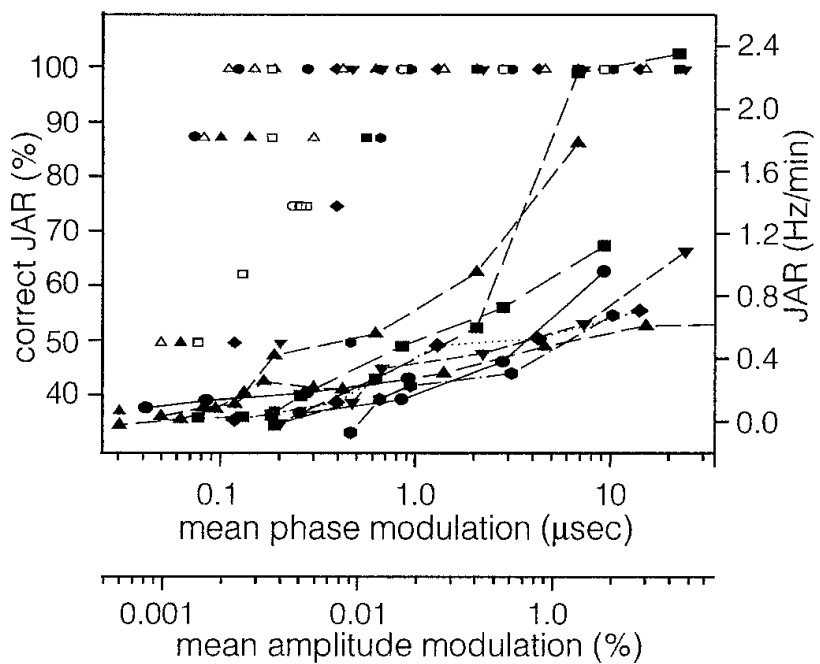

Figure 4. $A$, EOD frequencies during JAR with progressively smaller $\left|\mathrm{S}_{2}\right| /\left|\mathrm{S}_{1}\right|$ ratios, which yielded smaller phase and amplitude modulations. The frequency difference between $S_{1}$ and $S_{2}$ was alternated between +2 and $-2 \mathrm{~Hz}$ every $30 \mathrm{sec}$ at the dotted lines. Mean phase modulation (numbers on top left corners) was estimated as described in Materials and Methods. All traces presented show a 100\% correct response. B, Collective data from eight individuals. Different symbols represent different individuals. Unconnected symbols for percentage of correct responses, connected symbols for magnitude of JAR. The second abscissa expresses amplitude modulations inferred from the estimated ratio of $\left|S_{2}\right| /\left|S_{1}\right|$.

modulation was $<1 \mu \mathrm{sec}, 16$ of 36 behavioral sessions yielded $100 \%$ correct responses. Even when the mean phase modulation was $<200$ nsec, 10 of 16 sessions showed $\geq 87.5 \%$ correct responses. JAR requires not only phase modulation but also concurrent amplitude modulation (Kawasaki, 1993a). Because the magnitude of local amplitude modulation is a strict function of 


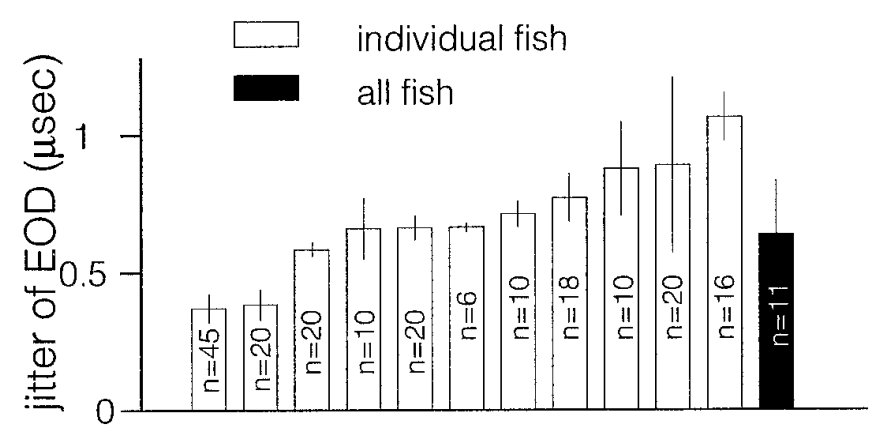

Figure 5. Jitter of EODs. For each fish, EODs were repeatedly sampled for $10 \sec (n=6-45)$. Jitter $(\sigma)$ was computed for each $10 \mathrm{sec}$ session, and the mean $\pm \mathrm{SD}$ of $\sigma$ is plotted (all but the right-most column). There was no apparent correlation between the size of fish and stability. The bar at far right represents data from all individuals showing mean $\pm \mathrm{SD}$ of 11 bars on the left.

$\left|S_{2}\right| /\left|S_{1}\right|$, one can infer corresponding magnitude of amplitude modulation. The corresponding amplitude modulation at the behavioral threshold was $0.02 \%$.

\section{Jitter of EODs}

Jitter of EODs was measured from 11 individuals. As shown in Figure 5 , the jitter of EOD was generally $<1 \mu$ sec $(0.65 \pm 0.19$ $\mu$ sec, $n=11$ ). For two particularly stable individuals, jitter was $0.38 \pm 0.05 \mu \mathrm{sec}$.

\section{JAR with artificial jitter in $\mathrm{S}_{\mathbf{1}}$}

Is this high degree of stability of EOD necessary for the detection of small phase differences for the JAR? We artificially introduced jitter in $S_{1}$ and measured JAR in curarized fish using the same experimental procedure as in Figure 4, except that $S_{1}$ was artificially jittered. Under this condition, all PLNs should experience the introduced jitter commonly; thus, no phase differences should be created between PLNs by jitter. The magnitude of JAR and percentage of correct responses were compared in three conditions, i.e., $\sigma_{\mathrm{S} 1}=0$ (no jitter), $\sigma_{\mathrm{S} 1}=20 \mu \mathrm{sec}$, and $\sigma_{\mathrm{S} 1}=60 \mu \mathrm{sec}$, with progressively smaller amplitudes of $S_{2}$ and, thus, of differential phase modulation. As shown in Figure 6, JARs occurred with comparable magnitudes and accuracy even when differential phase modulation was in the nanosecond range and $\mathrm{S}_{1}$ contained a large jitter that was 100 -fold larger than the phase differences.

\section{DISCUSSION}

\section{Accurate representation of temporal information by PLNs}

A major result of this study is that phase, i.e., zerocrossing times of electrosensory signals at electroreceptors, is represented by firing times of individual PLNs in the ELL with an accuracy of a few microseconds (Fig. 2). Corresponding vector strength, a measure of the degree of phase locking as defined by Goldberg and Brown (1969), for the mean jitter $(5.6 \mu \mathrm{sec})$ in this study is 0.9998 . This value is far greater than that typically found in the VIIIth nerve of the mammalian auditory system (Anderson et al., 1970). The highest degree of neural phase locking in the vertebrate auditory system is found in the barn owl, which uses small interaural time differences for sound localization (Knudsen et al., 1979). Neurons in the nucleus of magnocellularis of the barn owl show vector strength from 0.5 to 0.7 at low frequencies, which corresponds to a jitter of several tens of microseconds (Sullivan and Konishi, 1984; Carr and Konishi, 1990). Jitter measurement
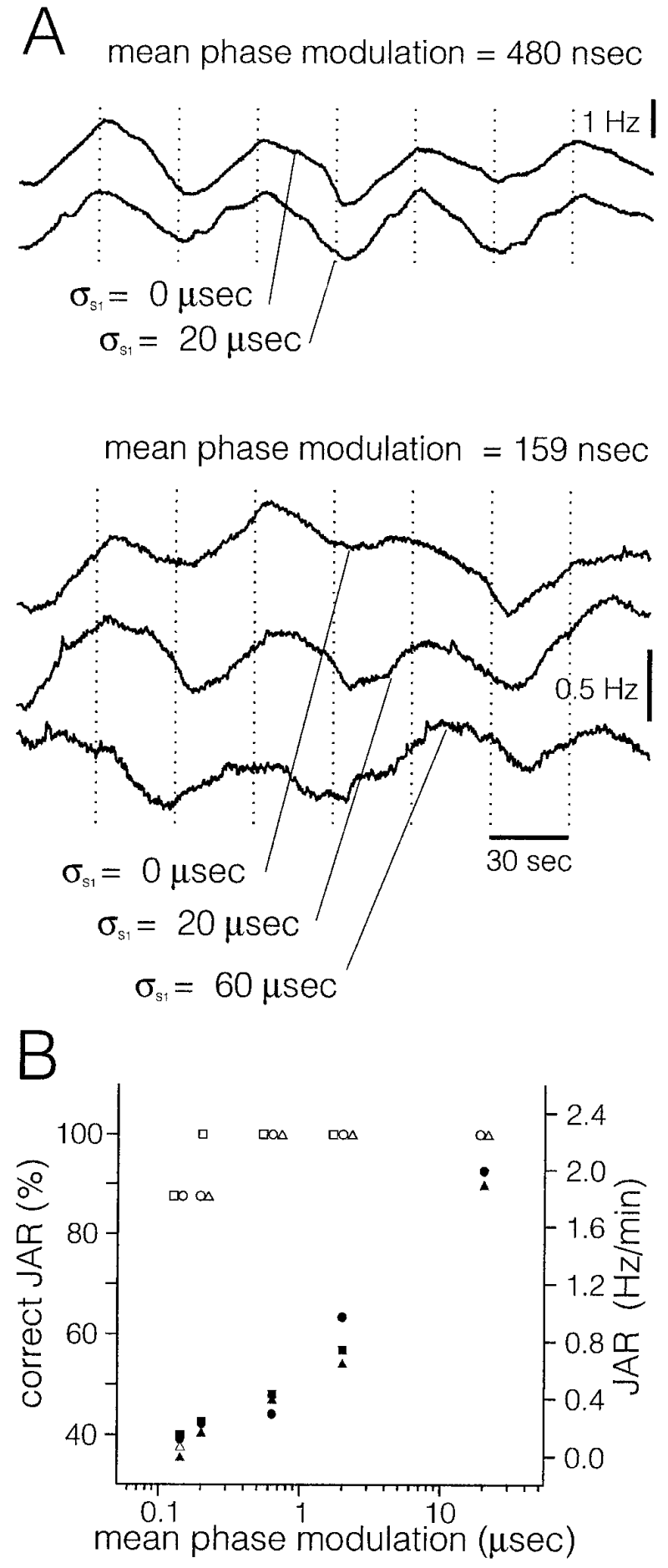

Figure 6. JAR occurred with small phase differences even when $\mathrm{S}_{1}$ was strongly jittered in phase. $A$, Top, Frequency traces showing JARs evoked with a mean phase difference of $480 \mathrm{nsec}$. The addition of jitter to $\mathrm{S}_{1}\left(\sigma_{\mathrm{S} 1}\right.$ $=20 \mu \mathrm{sec}$ ) had no significant effect on JAR. Bottom, With $159 \mathrm{nsec}$ of mean phase modulation, a marginal JAR was induced (top). Introduction of strong jitter (middle: $\sigma_{\mathrm{S} 1}=20 \mu \mathrm{sec}$; bottom: $\sigma_{\mathrm{S} 1}=60 \mu \mathrm{sec}$ ) did not disrupt the JARs. Data are from one individual. Two other individuals tested showed similar results. $B$, Percentage of correct responses and magnitude of JAR with progressively smaller phase differences with jittered $\mathrm{S}_{1}\left(\sigma_{\mathrm{S} 1}=20 \mu \mathrm{sec}\right)$. The addition of jitter in $\mathrm{S}_{1}$ had no significant effects over a wide range of magnitude of phase modulation (compare Fig. 4B). Open symbols show percentage of correct responses, filled symbols for JAR. Different shapes of symbols represent data from different individuals. 
in phase-locked neurons of an electric fish, Eigenmannia, (Rose and Heiligenberg, 1985b; Carr et al., 1986a) shows somewhat larger values than those found here in Gymnarchus. The temporal accuracy of the PLNs of Gymnarchus roughly corresponds to the sensitivity of differential-phase-sensitive neurons in the ELL, which are presumed to receive direct inputs from PLNs (Kawasaki and Guo, 1996).

\section{Temporal sensitivity expressed in the JAR}

Gymnarchus always showed $100 \%$ correct JARs when mean phase modulation was $>1 \mu \mathrm{sec}$ (Fig. 4). Distinctive JARs were often recorded with mean phase modulation of a few hundred nanoseconds. The behavioral sensitivity to microsecond time disparities is not unique to Gymnarchus; similar or even better temporal sensitivity has been shown in the electrosensory system of a gymnotiform electric fish as well as other vertebrate auditory systems (Rabizza and Masterton, 1972; Brown et al., 1978; Knudsen et al., 1979; Simmons et al., 1983). Despite independent evolution of the electrosensory and electrogeneric systems (Lauder and Liem, 1983), gymnotiform electric fish Eigenmannia performs JARs that are very similar to those of Gymnarchus (Bullock et al., 1975) using identical computational algorithms, consisting of multiple steps of amplitude and phase processing (Bullock et al., 1975; Heiligenberg et al., 1978; Heiligenberg and Bastian, 1980b; Heiligenberg, 1991; Kawasaki, 1993a, 1996). Eigenmannia exhibits comparable phase sensitivity to that found in this study (Rose and Heiligenberg, 1985b; Carr et al., 1986a). The brain structures for phase comparison, however, differ in these two species of electric fishes. The phase comparison circuit exists in a midbrain structure, the torus semicircularis, in Eigenmannia (Rose and Heiligenberg, 1985a, 1986a,b; Carr et al., 1986b) but in a hindbrain structure, the ELL, in Gymnarchus (Kawasaki and Guo, 1996).

\section{A possible role of the differential-phase system in electrolocation}

In both Gymnarchus and Eigenmannia, a larger difference in frequency (more than tens of Hertz) between a fish's own and a neighbor's EOD does not induce JAR and, correspondingly, has little effect on electrolocation performance (Heiligenberg, 1973, 1975) - frequency differences of a few Hertz have the strongest effects on the JAR and interference effects on the electrolocation performance. From those observations, one can speculate that the functional significance of the JAR is to increase the beat frequency resulting from EODs of two fish by mutually creating a larger frequency difference (Bullock et al., 1972; Heiligenberg, 1977, 1991). Although both Gymnarchus and Eigenmannia shift the discharge frequency correctly in their JAR when phase differences are small, the magnitude of the JARs in such a condition is too small to create a large frequency difference. Thus, the small and slow JARs near the threshold conditions may not serve to improve electrolocation performances.

This highly sensitive differential-phase system, however, may have a functional significance for electrolocation itself while no jamming signals exist. As reported by Kawasaki and Guo (1996, see Fig. $12 G$ ), PLNs in the ELL give rise to action potentials with different latencies for different stimulus amplitudes. Modulation of stimulus amplitude by $10 \%$ can result in a latency shift of PLNs' spiking by several tens of microseconds. The accuracy of PLN spiking found in this study implies that an electrolocation target may differentially shift the firing times of PLNs by local amplitude modulation, and differential-phase information associated with the target may be available to the differential-phase-sensitive neurons in the ELL. Amplitude-dependent latency shift in phasecoding afferent fibers, the T-afferents, of Eigenmannia has been examined but has not been measured with sufficient accuracy (Bullock and Chichibu, 1965; Heiligenberg and Partridge, 1981) to test a possible role of the phase system in electrolocation. Thus, the differential-phase system of Gymnarchus and Eigenmannia may be of multifunctional serving for both electrolocation and JAR in both genera. The role of the differential-phase system in electrolocation needs to be examined by recording from differential-phase-sensitive neurons while presenting electrolocation targets in both genera. The same experiments are needed in Sternopygus, which does not perform JAR (Matsubara, 1982; Rose et al., 1987), and in pulse gymnotiform species that do not use phase information for their JARs (Heiligenberg, 1980). Such comparative analysis should provide insight into the evolutionary origin of the differential-phase system.

Electrolocation objects with capacitance should also evoke local phase shifts. Involvement of phase-sensitive systems for detection of capacitive objects has been implicated in other electric fish species (Meyer, 1982; von der Emde and Ringer, 1992; von der Emde and Bell, 1994).

\section{Dynamic responses of PLNs}

As shown in Figure $3 A-C$, PLNs follow the phase modulation of a stimulus up to several tens of Hertz and thus could encode the rapid changes in electrosensory signals. If each PLN action potential were a response strictly to a past cycle of stimulus with a fixed latency, however, the dynamic phase responses of PLNs would be flat over higher frequencies up to the signal's carrier frequency. The sharp decline of gain spectrum at higher frequencies (Fig. 3C) suggests that PLNs and their associated electroreceptors may have a resonance property by which a firing time of a particular action potential is affected by the past several cycles. In such conditions, response phases to a rapid dynamic phase modulation may differ from neuron to neuron, and this may introduce phase differences between their responses. To test this notion, simultaneous recordings from pairs of PLNs were performed (Fig. 3D,E). They revealed that PLN phases are commonly modulated by phase modulations of stimuli even with a high-frequency phase modulation. Thus, little differential modulation between these neurons is introduced as long as the neurons are commonly driven by a single signal source.

\section{Stability of EODs}

EOD jitter measured in this study confirms the measurement of EOD jitter by Bullock et al. (1975). The frequency "singing" described in Bullock et al. (1975), however, was not observed, and all individuals showed stable EOD in a given measurement period (10 sec).

\section{JAR under $\mathbf{S}_{\mathbf{1}}$ with artificial jitter}

The rapid dynamics of phase responses in the PLNs (Fig. 3) implied that differential-phase information would be preserved even when a carrier signal (EOD or $\mathrm{S}_{1}$ ) contained jitter. This hypothesis was tested by adding artificial jitter in $S_{1}$ (Fig. 6). The experiment demonstrated that Gymnarchus performs normal JARs even when unnaturally large jitter $(\sigma=20-60 \mu \mathrm{sec})$ is introduced in $S_{1}$ and a minimal phase difference that is only a small fraction of the jitter was available to the fish. Thus, phase stability in the EOD is not required to detect small phase differences. 


\section{Emergence of the behavioral accuracy}

There are 10- to 100-fold differences in temporal accuracy expressed in the behavior (JAR) and in the differential-phasesensitive neurons in the ELL of Gymnarchus. A possible explanation of the difference is a spatial averaging of temporal information by a large number of differential-phase-sensitive neurons. The significance of the spatial averaging of electrosensory signal for the JAR of Eigenmannia is demonstrated behaviorally (Rose and Heiligenberg, 1985b), and a neuronal correlate of the behavioral accuracy is found in a premotor nucleus, the prepacemaker nucleus of Eigenmannia (Kawasaki et al., 1988a,b). In Gymnarchus, neuronal circuits for JAR beyond the ELL (Kawasaki and Guo, 1996) that possibly perform such spatial averaging have not yet been studied.

\section{REFERENCES}

Anderson DJ, Rose JE, Hind JE, Brugge JF (1970) Temporal position of discharges in single auditory nerve fibers within the cycle of a sine-wave stimulus: frequency and intensity effects. J Acoust Soc Am 49:1131-1139.

Bass AH, Hopkins CD (1982) Comparative aspects of brain organization of an African "wave" electric fish, Gymnarchus niloticus. J Morphol 174:313-334.

Brown CH, Beecher MD, Moody DB, Stebbins WC (1978) Localization of primate calls by Old World monkey. Science 201:753-754.

Bullock TH, Chichibu S (1965) Further analysis of sensory coding in electroreceptors of electric fish. Proc Natl Acad Sci USA 54:422-429.

Bullock TH, Behrend K, Heiligenberg W (1975) Comparison of the jamming avoidance responses in Gymnotoid and Gymnarchid electric fish: a case of convergent evolution of behavior and its sensory basis. J Comp Physiol 103:97-121.

Bullock TH, Hamstra RH, Scheich H (1972) The jamming avoidance response of high frequency electric fish. I. General features. J Comp Physiol 77:1-22.

Carr CE, Konishi M (1990) A circuit for detection of interaural time differences in the brain stem of the barn owl. J Neurosci 10:3227-3246.

Carr CE, Heiligenberg W, Rose GJ (1986a) A time-comparison circuit in the electric fish midbrain. I. Behavior and physiology. J Neurosci 6:107-119.

Carr CE, Maler L, Taylor B (1986b) A time-comparison circuit in the electric fish midbrain. II. Functional morphology. J Neurosci 6:1372-1383.

Goldberg JM, Brown PB (1969) Response of binaural neurons of dog superior olivary complex to dichotic tonal stimuli: some physiological mechanisms of sound localization. J Neurophysiol 32:613-636.

Heiligenberg W (1973) Electrolocation of objects in the electric fish Eigenmannia (Rhamphichthydae, Gymotoidei). J Comp Physiol 87:137-164.

Heiligenberg W (1975) Electrolocation and jamming avoidance in the electric fish Gymnarchus niloticus (Gymnarchidae, Mormyriformes). J Comp Physiol 103:55-67.

Heiligenberg W (1977) Principles of electrolocation and jamming avoidance in electric fish: a neuroethological approach. New York: Springer.

Heiligenberg W (1980) The evaluation of electroreceptive feedback in a Gymnotoid fish with pulse-type electric organ discharges. J Comp Physiol [A] 138:173-185.

Heiligenberg W (1991) Neural nets in electric fish. Cambridge: MIT.

Heiligenberg W, Bastian J (1980a) The control of Eigenmannia's pacemaker by distributed evaluation of electroreceptive afferences. J Comp Physiol [A] 136:113-133.

Heiligenberg W, Bastian J (1980b) Species specificity of electric organ discharges in sympatric Gymnotoid fish of the Rio Negro. Acta Biol Venez 10:187-203.

Heiligenberg W, Partridge BL (1981) How electroreceptors encode JAR-eliciting stimulus regimes: reading trajectories in a phaseamplitude plane. J Comp Physiol 142:295-308.

Heiligenberg W, Rose G (1985) Phase and amplitude computations in the midbrain of an electric fish: intracellular studies of neurons participating in the jamming avoidance response of Eigenmannia. J Neurosci 5:515-531.
Heiligenberg W, Baker C, Matsubara J (1978) The jamming avoidance response in Eigenmannia revisited: the structure of a neuronal democracy. J Comp Physiol 127:267-286.

Kawasaki M (1993a) Independently evolved jamming avoidance responses employ identical computational algorithms: a behavioral study of the African electric fish, Gymnarchus niloticus. J Comp Physiol [A] 173:9-22.

Kawasaki M (1993b) Temporal hyperacuity in the gymnotiform electric fish, Eigenmannia. Am Zool 33:86-93.

Kawasaki M (1994) The African wave-type electric fish, Gymnarchus niloticus, lacks corollary discharge mechanisms for electrosensory gating. J Comp Physiol [A] 174:133-144.

Kawasaki M (1996) Comparative analysis of the jamming avoidance response in African and South American wave-type fishes. Biol Bull 191:103-108.

Kawasaki M, Guo Y-X (1996) Neuronal circuitry for comparison of timing in the electrosensory lateral line lobe of an African wave-type electric fish, Gymnarchus niloticus. J Neurosci 16:380-391.

Kawasaki M, Maler L, Rose GJ, Heiligenberg W (1988a) Anatomical and functional organization of the prepacemaker nucleus in gymnotiform electric fish: the accommodation of two behaviors in one nucleus. J Comp Neurol 276:113-131.

Kawasaki M, Rose GJ, Heiligenberg W (1988b) Temporal hyperacuity in single neurons of electric fish. Nature 336:173-176.

Knudsen EI, Blasdel GG, Konishi M (1979) Sound localization by the barn owl (Tyto alba). J Comp Physiol 133:13-21.

Lauder GV, Liem KF (1983) Patterns of diversity and evolution in rayfinned fishes. In: Fish neurobiology (Northcutt RG, Davis RE, eds), pp 1-24. Ann Arbor, MI: University of Michigan.

Lissmann HW, Machin KE (1958) The mechanism of object location in Gymnarchus niloticus and similar fish. J Exp Biol 35:451-486.

Matsubara J (1982) Physiological cell types in the electrosensory lateral line lobe of weakly electric fish: neural correlates of electrolocation under jamming. J Comp Physiol [A] 149:339-351.

Meyer HJ (1982) Behavioral responses of weakly electric fish to complex impedances. J Comp Physiol [A] 145:459-470.

Mills AW (1972) Auditory localization. In: Foundation of modern auditory theory (Tobias JV, ed), pp 303-348. New York: Academic.

Rabizza RJ, Masterton RB (1972) Contribution of neocortex to sound localization in the opossum (Dedelphis virginiana). J Neurophysiol 35:344-356.

Rose GJ, Heiligenberg W (1985a) Structure and function of electrosensory neurons in the torus semicircularis of Eigenmannia: morphological correlates of phase and amplitude sensitivity. J Neurosci 5:2269-2280.

Rose GJ, Heiligenberg W (1985b) Temporal hyperacuity in the electric sense of fish. Nature 318:178-180.

Rose GJ, Heiligenberg W (1986a) Limits of phase and amplitude sensitivity in the torus semicircularis of Eigenmannia. J Comp Physiol [A] 159:813-822.

Rose GJ, Heiligenberg W (1986b) Neural coding of difference frequencies in the midbrain of the electric fish Eigenmannia: reading the sense of rotation in an amplitude-phase plane. J Comp Physiol [A] 158:613-624.

Rose GJ, Keller CH, Heiligenberg W (1987) “Ancestral” neural mechanisms of electrolocation suggest a substrate for the evolution of the jamming avoidance response. J Comp Physiol [A] 160:491-500.

Simmons JA, Kick SA, Lawrence BD, Hale C, Brad C, Escudie B (1983) Acuity of horizontal angle discrimination by the echolocating bat, Eptesicus fuscus. J Comp Physiol [A] 153:321-330.

Sullivan WE, Konishi M (1984) Segregation of stimulus phase and intensity coding in the cochlea nucleus of the barn owl. J Neurosci 4:1787-1799.

Szabo T (1962) Spontaneous electrical activity of cutaneous receptors in Mormyrids. Nature 194:600-601.

von der Emde G, Bell CC (1994) Responses of cells in the mormyrid electrosensory lobe to EODs with distorted wave-forms: implications for capacitance detection. J Comp Physiol [A] 175:83-93.

von der Emde G, Ringer T (1992) Electrolocation of capacitative objects in four species of pulse-type weakly electric fish. I. Discrimination Performance. Ethology 91:326-338. 